\title{
Extracorporeal shockwave therapy for the treatment of lower limb intermittent claudication: study protocol for a randomised controlled trial (the SHOCKWAVE 1 trial)
}

\author{
Thomas Cayton, Amy E. Harwood, George E. Smith, Joshua P. Totty*, Daniel Carradice and lan C. Chetter
}

\begin{abstract}
Background: Peripheral arterial disease (PAD) has a population prevalence of $4.6 \%$ with intermittent claudication (IC) presenting as one of the earliest and most common symptoms. PAD has detrimental effects on patients' walking ability in terms of maximum walking distance (MWD) and pain-free walking distance (PFWD). Research has suggested extracorporeal shockwave therapy (ESWT) may induce angiogenesis in treated tissue; therefore, our objective is to assess the tolerability and efficacy of ESWT as a novel treatment of intermittent claudication.

Methods/design: Patients with unilateral claudication will be randomised to receive either ESWT (PiezoWave 2 shockwave system) or sham treatment to the calf muscle bulk three times per week for 3 weeks. All patients are blinded to treatment group, and all assessments will be performed by a masked assessor. Treatment tolerability using a visual analogue scale, ankle-brachial pressure index, MWD, PFWD and safety will all be formally assessed as outcome measures at baseline and at 4, 8 and 12 weeks follow-up.

Discussion: This trial will be the first of its kind in terms of methodology in relation to ESWT for intermittent claudication. A double-masked randomised controlled trial will provide useful information about the potential for the use of ESWT as a non-invasive treatment option and the need for further robust research.
\end{abstract}

Trial registration: ClinicalTrials.gov, NCT02652078. Registered on 17 October 2014.

\section{Introduction}

Peripheral arterial disease (PAD) is common in western society with a prevalence estimated at $20 \%$ of those aged over 60 [1]. Intermittent claudication (IC), a manifestation of PAD in the lower limbs, causes sufferers to experience cramping pain in the affected muscles, most commonly the calves, which occurs after walking a short distance and increases in intensity until the person stops, where the pain then gradually subsides. PAD can be limiting or occasionally debilitating and has been shown to have considerable deleterious effects on patients' quality of life [2].

* Correspondence: joshua.totty@hyms.ac.uk

Academic Vascular Surgery Unit, Hull and East Yorkshire Hospitals NHS Trust, Hull Royal Infirmary, Anlaby Road, Hull HU3 2JZ, UK
There is a 15-fold increase in mortality secondary to cardiovascular disease in symptomatic patients with severe large vessel PAD; therefore, treatment is based around minimising cardiovascular risk factors and reducing risk of disease progression [3]. With conservative management strategies a proportion of patients will remain with stable symptoms, whilst a minority will deteriorate and require intervention in the form of an endovascular or open vascular surgical procedure.

The physiological negative effects caused by PAD are hypothesised to be due in part to recurrent episodes of ischaemia-reperfusion injury (IRI), which occurs as claudicants exercise (ischaemia) and rest (reperfusion). IRI causes progression of atherosclerosis, as well as endothelial dysfunction and end organ damage [4-6]. During 
ischaemic episodes and subsequent reperfusion, leucocytes become activated, resulting in release of proteolytic enzymes, cytokines and reactive oxygen species $[7,8]$.

\section{Background}

A shockwave is described as a transient short-term acoustic pulse with a high peak pressure. This waveform generation and rise to peak pressure occur within nanoseconds [9]. Biological effects of shockwaves on human tissue were first documented during World War II when severe lung injuries secondary to water bombs were noted within casualties who did not display any evidence of external injury [10]. The medicinal use of high energy extracorporeal shockwaves did not develop until the 1980s, when they were targeted to fragment urinary stones (lithotripsy); results led to this technique becoming the gold standard treatment option for urolithiasis [9]. From this point the developing use of extracorporeal shockwave therapy (ESWT) followed an interesting course which started after an incidental finding of iliac bone thickening in patients undergoing lithotripsy, which proposed the potential osteoblastic effect of shockwave therapy [11]. Researchers confirmed these observations through a number of clinical trials using shockwave therapy on delayed non-union fractures [12-14]. Observational improvements to soft tissue wounds overlying these fractures treated with ESWT then encouraged research into the benefits of shockwave therapy in hard-to-heal chronic soft tissue wounds [15].

The exact mechanism of action of ESWT on soft tissue is not fully understood, although the principle of mechanotransduction is thought to be responsible for the biological changes that take place. Mechanotransduction is the transfer of mechanical stimuli into chemical signals; this takes place at a cellular level where mechanosensory components within the cell wall are stimulated, which activates gene expression of growth factors and cytokines as well as cell proliferation or differentiation [16]. The mechanical force is a secondary effect of cavitation which can be visualised in water by the creation of cavitation bubbles that then emit secondary shockwaves. This physical force can also be seen when shockwaves are directed over a thin aluminium foil, which show the effect of cavitation bubbles collapsing on or near the foil [17].

Increased expression of growth factors such as endothelial nitric oxide synthase (eNOS) and vascular endothelial growth factors (VEGFs) have been demonstrated following treatment of soft tissues with ESWT during various studies both in vivo and in vitro. However, the majority of this evidence is from animal studies, and further investigation is required [18-21].

Due to these findings, further applications of ESWT are beginning to be explored. One such application included the use of ESWT to treat ischaemic heart disease where focussed shockwaves were directed at ischaemic areas of myocardium. This demonstrated some positive findings with notable results including improvement in left ventricular ejection fraction (LVEF), ventricular wall thickening, reduced symptoms, reduced nitroglycerine use and again increased upregulation of growth factors and angiogenesis [22]. A further use has potentially been found with small trials commencing in ESWT for peripheral vascular disease, which has led to the development of this current protocol.

\section{Summary of current evidence}

To date, four studies have investigated ESWT in claudicants (Table 1). They include one randomised control trial of 22 patients with 12 patients receiving treatment and 10 receiving a sham control. This study targeted ESWT at a stenotic plaque lesion within lower limb arteries and demonstrated significant reduction in degree of arterial stenosis, significant improvement in pain-free walking distance (PFWD) and reduction in pain severity. These findings were also coupled with a significant reduction in Fontaine staging [23].

Another study was a small prospective non-randomised pilot study of 10 patients which aimed to prove safety; its primary end point was observation of adverse events. This study found no adverse events secondary to shockwave therapy. The secondary end point was a measure of tissue perfusion using scintography, laser Doppler and measurement of transcutaneous oxygen tension $\left(\mathrm{TcPO}_{2}\right)$. The use of scintography demonstrated significantly improved tissue perfusion in the dorsum of the foot; however, perfusion was unchanged in the lower leg region. Maximum $\mathrm{TcPO}_{2}$ increased significantly in both the calf region and dorsum of the foot. Skin perfusion pressure measured using the laser Doppler showed increases in both regions, but neither was significant [24].

Another prospective study of 32 patients underwent EWST for critical limb ischaemia with both rest pain and localised distal necrosis. Outcomes included PFWD, visual analogue scale (VAS) for pain, laser Doppler skin perfusion, partial pressure of oxygen and partial pressure of carbon dioxide. All of these outcomes demonstrated significantly improved measurements [25].

Finally a further two prospective non-randomised trials by Serizawa et al. with a total of 6 patients and then 12 patients with Fontaine stage II PAD were treated with ESWT and demonstrated significantly improved maximum walking distance, shorter recovery time and improved walking impairment questionnaire scores [26].

\section{Aims and objectives}

This study aims to compare the effects of extracorporeal shockwave therapy (ESWT) to the use of a sham control group on walking distances in subjects with lower limb intermittent calf claudication. 
Table 1 Human studies related to ESWT for peripheral arterial disease

\begin{tabular}{|c|c|c|c|c|c|}
\hline Study/author(s) & Type & Sample size & Methods & Significant results & Limitations \\
\hline $\begin{array}{l}\text { Ciccone MM, } \\
\text { Notarnicola A [23] }\end{array}$ & $\begin{array}{l}\text { Randomised } \\
\text { control trial }\end{array}$ & $\begin{array}{l}N=22 \\
\text { cases }=12, \\
\text { controls }=10\end{array}$ & $\begin{array}{l}4 \text { sessions at } 1 \text { weekly intervals, } \\
2000 \text { impulses, } 0.03 \mathrm{~mJ} / \mathrm{mm}^{2} \text {. } \\
\text { Aimed at stenotic lesion }\end{array}$ & $\begin{array}{l}\text { Increased pain-free walking distance } \\
\text { and reduction in pain severity. } \\
\text { Reduction in degree of arterial } \\
\text { stenosis. Reduced Fontaine staging }\end{array}$ & $\begin{array}{l}\text { Small sample size, } \\
\text { short-term follow-up }\end{array}$ \\
\hline $\begin{array}{l}\text { Tara S, } \\
\text { Miyamoto M [24] }\end{array}$ & $\begin{array}{l}\text { Prospective } \\
\text { non-randomised } \\
\text { pilot study }\end{array}$ & $N=10$ & $\begin{array}{l}6 \text { sessions ( } 3 \text { times per week } \\
\text { for } 2 \text { weeks). } \\
6 \text { spots per session ( } 300 \text { impulses/ } \\
\text { spot) } 0.11-0.12 \mathrm{~cm} / \mathrm{mm}^{2}\end{array}$ & $\begin{array}{l}\text { No adverse events. } \\
\text { Improved transcutaneous oxygen } \\
\text { tension }\left(\mathrm{TCPO}_{2}\right) \text { in both calf and } \\
\text { dorsum of foot. Scintography } \\
\text { demonstrated improved perfusion } \\
\text { in dorsum of foot only }\end{array}$ & $\begin{array}{l}\text { Small sample size, } \\
\text { non-randomised, } \\
\text { short-term follow-up }\end{array}$ \\
\hline $\begin{array}{l}\text { Belcaro G, } \\
\text { Cesarone MR [25] }\end{array}$ & $\begin{array}{l}\text { Prospective } \\
\text { non- randomised }\end{array}$ & $N=32$ & $\begin{array}{l}6 \text { sessions ( } 3 \text { times per week } \\
\text { for } 2 \text { weeks) } 1000 \text { impulses } \\
\text { per session, } 0.08-0.43 \mathrm{~mJ} / \mathrm{mm}^{2}\end{array}$ & $\begin{array}{l}\text { Improved pain-free walking } \\
\text { distance, analogue scale for pain, } \\
\text { laser Doppler skin perfusion, partial } \\
\text { pressure of oxygen and decreased } \\
\text { partial pressure of carbon dioxide. } \\
\text { Reduced ORACLE score }\end{array}$ & $\begin{array}{l}\text { Small sample size, } \\
\text { non-randomised } \\
\text { short-term follow-up }\end{array}$ \\
\hline $\begin{array}{l}\text { Serizawa F, } \\
\text { Ito K [26] }\end{array}$ & $\begin{array}{l}\text { Prospective } \\
\text { non-randomised }\end{array}$ & $N=12$ & $\begin{array}{l}9 \text { sessions ( } 3 \text { times per week } \\
\text { for } 3 \text { weeks) } 40 \text { spots per session } \\
\text { with } 200 \mathrm{impulses} / \mathrm{spot} 0.1 \mathrm{~mJ} / \mathrm{mm}^{2}\end{array}$ & $\begin{array}{l}\text { Improved maximum walking } \\
\text { distance, walking impairment } \\
\text { questionnaire scores and } \\
\text { shorter recovery time }\end{array}$ & $\begin{array}{l}\text { Small sample size, } \\
\text { non-randomised, } \\
\text { short-term follow-up }\end{array}$ \\
\hline $\begin{array}{l}\text { Serizawa F, } \\
\text { Ito K [26] }\end{array}$ & $\begin{array}{l}\text { Prospective } \\
\text { non-randomised }\end{array}$ & $N=6$ & $\begin{array}{l}6 \text { sessions ( } 3 \text { times in first week } \\
\text { (days } 1,3 \text { and } 5 \text { ) and } 3 \text { sessions } \\
\text { in week } 5 \text { (days } 29,31,33 \text { ) } 40 \\
\text { spots per session with } 200 \\
\text { impulses/spot } 0.1 \mathrm{~mJ} / \mathrm{mm}^{2}\end{array}$ & $\begin{array}{l}\text { The maximum walking distance } \\
\text { was significantly increased at } \\
4 \text { weeks and } 8 \text { weeks follow-up }\end{array}$ & $\begin{array}{l}\text { Small sample size, } \\
\text { non-randomised, } \\
\text { short-term follow-up }\end{array}$ \\
\hline
\end{tabular}

Data captured in italic are significant outcome for measures in the trial protocol

\section{Primary outcome}

The primary outcome is the effect of extracorporeal shockwave therapy in comparison to sham treatment on the maximum walking distance (MWD) - the distance covered before the subject is unable to continue - at baseline and at 4 weeks, 8 weeks and 12 weeks post therapy.

\section{Secondary outcomes}

The secondary outcomes are as follows:

- Claudication distance, i.e. distance covered prior to onset of any symptoms, at baseline and at 4 weeks, 8 weeks and 12 weeks post therapy

- Ankle-brachial pressure index (ABPI), measured at baseline and at 4 weeks, 8 weeks and 12 weeks post therapy

- Patient reported walking distance at baseline, 4 weeks, 8 weeks and 12 weeks post therapy

- Quality of life as measured by Short Form 36 questionnaire (SF-36) at baseline, 4 weeks, 8 weeks and 12 weeks post therapy

- Quality of life as measured by the EuroQol 5 domains questionnaire (EQ-5D) at baseline, 4 weeks, 8 weeks and 12 weeks post therapy

- Subject tolerance of treatment, measured by VAS after each individual treatment session

\section{Design}

This is a single-centre, double-blinded randomised controlled trial comparing extracorporeal shockwave therapy (ESWT) to sham treatment in the management of intermittent calf claudication.

\section{Setting}

The trial will be conducted in the Academic Vascular Surgical Unit of the Hull York Medical School, based at Hull Royal Infirmary (University Teaching Hospital). All procedures will be performed in an outpatient-based or day-case setting.

\section{Patient identification}

Patients will be recruited from vascular outpatient clinics and assessed for inclusion by the research team against the listed inclusion and exclusion criteria. In order to achieve target sample size, all patients identified as suffering from PAD will be screened for inclusion.

\section{Target population and recruitment Inclusion criteria}

Patients will be included who:

- Have unilateral or bilateral intermittent calf claudication (stable for the last 3 months)

- Are able to give written informed consent to participate in the study 
- Are $>18$ years old

- Are able to adhere to the protocol and attend all follow-up appointments

- Are currently receiving 'best medical therapy' antiplatelet and statin medication

\section{Exclusion criteria}

Patients will be excluded if they:

- Currently have a malignancy

- Are allergic to or intolerant of either antiplatelet medication or statin therapy

- Are pregnant (pregnancy test performed at screening if necessary)

- Have a metal implant near the treatment area

- Are taking anticoagulation medication (i.e. warfarin)

- Are diagnosed with a blood clotting disorder

\section{Screening and evaluation}

Patients identified as potential participants will undergo a screening interview.

Baseline data will be collected from all consenting participants. Data collected will include:

- Evaluation of compliance with inclusion and exclusion criteria

- Issue of Patient Information Sheet if not already issued

- Informed consent (24 hours later)

- Participant's name and address and, if used by the participant, mobile telephone number and email address (for the receipt of follow-up questionnaires) and date of birth

- Details on participant's general practitioner

- Demography and medical history

- Physical examination to include palpation of lower limb pulses

- Ankle-brachial pressure index (ABPI)

- Vital signs

- Assessment of any concurrent medications

- Pregnancy test if necessary

- Body mass index (BMI)

- Smoking history

- Baseline quality of life

Appropriately screened and consented individuals will be further assessed as follows:

1. Baseline visit

(a) Patient reported walking distance (PRD)

(b) Vital signs

(c) Quality of life
(d)Treadmill assessment to report intermittent claudication distance (ICD) and maximum walking distance (MWD)

For treadmill walking distances, ICD and MWD will be measured on a treadmill at $2.5 \mathrm{~km} / \mathrm{h}$ at a $10^{\circ}$ incline for up to 10 minutes. Participants will be asked to rest for 30 minutes prior to testing to ensure a resting state and negate effects of mobilising to the vascular laboratory.

Randomisation will occur at the end of the baseline visit via sealed envelope software to either active treatment or control group by a second investigator. The treatment will then proceed as follows:

(e) The second investigator will then perform ESWT therapy for the active group and sham treatment for the control group. Sham treatment will be delivered by setting the generator to off and replicating the sound of the active treatment via an audio recording emitted from the machine

(f) All patients will then complete a tolerability questionnaire for the ESWT treatment.

2. Weeks 1, 2, 3 visits

(a) Vital signs

(b)ESWT performed by second investigator with pulsewave generator on for active group and off for control group

(c) Patient to complete tolerability questionnaire for ESWT treatment

(d)PRD recorded

(e) Assessment of any concurrent medications

(f) Assessment of any adverse events

3. Week 4, 8, 12 visits
(a) PRD
(b) Vital signs
(c) Quality of life
(d)Treadmill assessment to report ICD and MWD
(e) Assessment of any concurrent medications
(f) Assessment of any adverse events

\section{Method/design}

\section{Trial treatments/study procedures}

Patients on the treatment arm of the trial will receive ESWT using the PiezoWave2 shockwave system, in accordance with the manufacturer's instructions and unchanged from its current clinical use at 100 impulses $0.1 \mathrm{~mJ} / \mathrm{mm}$ per $\mathrm{cm}^{2}$. Trained staff will provide all treatments, including sham treatment. Both local research and development and medical physics departments deem this device low risk and safe to use. The device will be targeted at the gastrocnemius muscles of the affected lower leg for several minutes at each treatment session. Participants in the control group will undergo 
an identical process as if treatment were being given but with the shockwaves not being administered and the sham treatment described above alternatively used.

1 shockwave session $=100$ impulses per $\mathrm{cm}^{2}$ covering two areas per affected leg, with one $(6 \mathrm{~cm} \times 5 \mathrm{~cm})$ on the medial head of the gastrocnemius and one $(6 \mathrm{~cm} \times$ $5 \mathrm{~cm}$ ) on the lateral head of the gastrocnemius. This calculates as 6000 impulses $/ 5 \mathrm{~Hz} / 0.1 \mathrm{~mJ} / \mathrm{mm}^{2}$ in total.

\section{Environmental conditions}

A designated clinical room in the vascular laboratory at Hull Royal Infirmary will be used for all examination and treatments. The device will be applied to the gastrocnemius muscle of the affected limb/s at two sites (medially and laterally). One course of treatment will consist of three separate treatment sessions per week on alternate days for 3 weeks.

\section{Outcome measurements}

\section{Primary outcome}

The primary outcome is the short-to-medium term effect of ESWT in comparison to sham treatment on MWD in patients suffering intermittent calf claudication at baseline, 4 weeks, 8 weeks and 12 weeks post therapy.

\section{Secondary outcomes}

The secondary outcomes are as follows:

- Claudication distance (CD) - the distance covered prior to onset of any symptoms - at baseline, 4 weeks, 8 weeks and 12 weeks post therapy

- Pre and post treatment ABPI at baseline, 4 weeks, 8 weeks and 12 weeks post therapy

- Patient reported walking distance (PRD) at baseline, 4 weeks, 8 weeks and 12 weeks post therapy

- Acceptability and tolerability of the treatment measured by VAS after each individual treatment session

- Control group patients will be asked to complete tolerability questionnaires as if they were receiving active treatment.

- Quality of life as measured by SF-36 at baseline, 4 weeks, 8 weeks and 12 weeks post therapy

- Quality of life as measured by the EQ-5D at baseline, 4 weeks, 8 weeks and 12 weeks post therapy

\section{Assessments}

Following completion of the consent procedure, baseline data will be collected as defined above. Outcome measurements will then be recorded according to the subsequent study visits. See Fig. 1 and Table 4.

Assessments prior to the first ESWT therapeutic session Assessments prior to the first EWST session are:

- PRD
- ABPI

- Claudication distance

- Maximum walking distance

- Quality of life questionnaires (SF-36 and EQ-5D)

\section{Assessments during all therapeutic treatments}

During all therapeutic treatments, we will assess:

- Pain rating of treatment using VAS

- Quality of life questionnaires (SF-36 and EQ-5D)

\section{Assessments during weeks 4, 8 and 12 follow-up appointments}

Assessments at weeks 4, 8 and 12 are:

- Tolerability questionnaire (week 4 only)

- PRD

- ABPI

- Claudication distance

- Maximum walking distance

- Quality of life questionnaires (SF-36 and EQ-5D)

\section{Concurrent medications}

The study will not influence clinical management of the patient; therefore, there are no restrictions of prescribed medication. Researchers will monitor concomitant medications taken by the patients at screening and during the entire study period.

\section{Adverse events}

Serious adverse events will be reported to the Sponsor and the National Research Ethics Service (NRES) recognised Research Ethics Committee (REC).

\section{The device}

The device being used in this trial is the PiezoWave 2 . Training and support are provided by Impact Medical, Liverpool, UK.

\section{Safety and tolerability}

The device is a CE-marked (CE 93/42/EEC) British Standards Institute, notified body 0086), commercially available device. Trained staff will manage the device and are responsible for applications of treatment. The facilities where participants will be assessed are fully equipped for all emergency situations.

\section{Safety reporting}

The safety definitions are given below.

Adverse event $(A E)$ : An $\mathrm{AE}$ is any untoward medical occurrence in a subject to whom a medicinal product has been administered as part of a research study, including occurrences which are not necessarily caused by or related to an investigational medicinal product (IMP). 


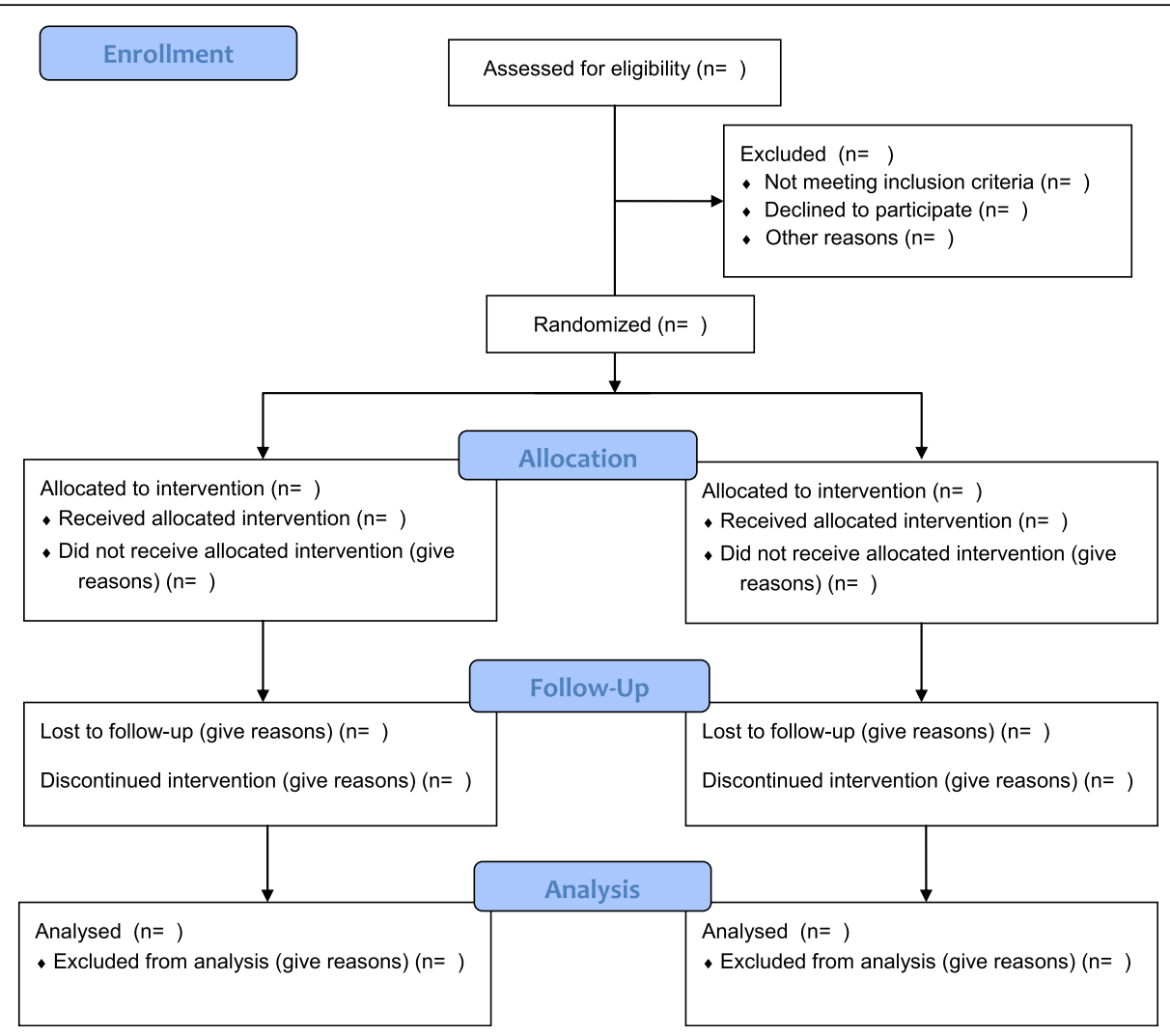

Fig. 1 Consolidated Standards of Reporting Trials (CONSORT) flow diagram

Adverse reaction (AR): An AR is any untoward and unintended response in a subject to an IMP which is related to any dose administered to that subject.

Important medical events that may not be immediately life-threatening or result in death or hospitalisation but may jeopardise the patient or may require intervention to prevent one of the other outcomes listed in the definition above should also be considered serious.

Serious adverse event (SAE): An SAE is an event not related or unlikely to be related to the IMP. An adverse event becomes serious if it:

- Results in death

- Is life-threatening

- Requires hospitalisation or prolongation of existing hospitalisation

- Results in persistent or significant disability or incapacity

- Is a congenital anomaly or birth defect

Serious adverse reaction (SAR): A SAR is a serious event which is suspected (possibly, probably or definitely) to be related to an IMP and expected for the IMP.

Suspected unexpected serious adverse reaction (SUSAR): A SUSAR is a serious event which is suspected (possibly, probably or definitely) to be related to an IMP and unexpected for the IMP, i.e. not previously documented in any of the IMP information (investigator brochure, Summary of Product Characteristics, patient information leaflet) or protocol on device deficiencies.

Device deficiency: A device deficiency is an inadequacy of a medical device with respect to its identity, quality, durability, reliability, safety or performance. All device deficiencies will be recorded and reported accordingly.

Anticipated and unanticipated serious adverse device effects (SADE/USADE): There are no known SADEs with this device; therefore, all SADEs are, by definition, unanticipated.

If a USADE is suspected, no additional patients will be enrolled until the cause of the event and its relationship to the study device have been determined. If the event is device-related, the following action will be necessary. If there is a specific type of patient who is vulnerable to the event, then the inclusion/exclusion criteria will be amended to exclude this 'at risk' group.

The protocol procedures may be revised including but not limited to the duration of therapy. The study may also be stopped. See Table 2 .

\section{Causality and adverse device effects}

The relationship of both AEs and SAEs to the study device will be determined as follows (Table 3). 
Table 2 Summary of adverse events

\begin{tabular}{|c|c|c|c|}
\hline $\begin{array}{l}\text { Adverse } \\
\text { events }\end{array}$ & $\begin{array}{l}\text { Non-device- } \\
\text { related }\end{array}$ & \multicolumn{2}{|l|}{ Device-related } \\
\hline Non-serious & $\begin{array}{l}\text { Adverse } \\
\text { event (AE) }\end{array}$ & \multicolumn{2}{|c|}{ Adverse device effect (ADE) } \\
\hline \multirow[t]{3}{*}{ Serious } & & \multicolumn{2}{|c|}{ Serious adverse device effect (SADE) } \\
\hline & & Anticipated & Unanticipated \\
\hline & & $\begin{array}{l}\text { Anticipated serious } \\
\text { adverse device } \\
\text { effect (ASADE) }\end{array}$ & $\begin{array}{l}\text { Unanticipated serious } \\
\text { adverse effect } \\
\text { (USADE) }\end{array}$ \\
\hline
\end{tabular}

The Investigator must endeavour to obtain sufficient information to determine the causality of the $\mathrm{AE}$ and must provide his/her opinion of the causal relationship between each $\mathrm{AE}$ and the study device. This may require instituting supplementary investigations of significant AEs based on their clinical judgement of the likely causative factors and/ or include seeking a further opinion from a specialist in the field of the AE.

\section{Adverse event and device deficiency reporting}

All AEs during the study will be reported on the Adverse Event page of the Case Report Form (CRF). If events are deemed serious, a separate Serious Adverse Event form will also be completed.

Device deficiencies during the study will be recorded on a dedicated form. The Principal Investigator will record the action taken to alleviate the AE/SAE. AEs may be recorded as ongoing at the end of the study, but SAEs will be followed up until resolved. Trained medical personnel will be accessed to act with appropriate diagnostic and therapeutic measures until the subject has recovered. SAEs will be reported to the Principal Investigator within 24 hours.

\section{Withdrawals and dropouts}

During the study, treatment may be discontinued for many reasons such as the occurrence of a disease, an $\mathrm{AE}$ that could interfere with the subject's evaluation or simply

Table 3 Causality of adverse events

\begin{tabular}{ll}
\hline Causality & Definition \\
\hline Almost certainly & $\begin{array}{l}\text { Starts within a time related to the operation } \\
\text { of the device, and } \\
\text { No obvious alternative medical explanation } \\
\text { Probably }\end{array}$ \\
$\begin{array}{l}\text { Starts within a time related to the operation } \\
\text { of the device, and } \\
\text { Cannot be reasonably explained by known } \\
\text { characteristics of the patient's clinical state } \\
\text { Possibly }\end{array}$ & $\begin{array}{l}\text { Starts within a time related to the operation } \\
\text { of the device, and } \\
\text { A causal relationship between the operation } \\
\text { of the device and the adverse event is at } \\
\text { least a reasonable possibility }\end{array}$ \\
& The AE is definitely not associated with the \\
Unrelated & study device
\end{tabular}

upon the subject's request to discontinue for any reason. Concurrent medical events that do not interfere with scheduled testing and that are judged by the Investigator to not have an effect on the outcome measures will not disqualify a subject from continuing in the study. If a subject is withdrawn from the study because of an $\mathrm{AE}$, treatment discontinuation must be explained on the Adverse Event page of the CRF.

Patients will be advised that they are free to withdraw from the study at any time for any reason or, if necessary, the Investigator may withdraw a subject from the study to protect the subject's health. The Investigator may withdraw a subject from the study if it is considered that the scientific, and therefore, ethical standards of the study are compromised. Patients may also be withdrawn for not complying with study procedures. The type and timing of the withdrawal for withdrawal will be fully recorded on the CRF. Should a patient choose to withdraw from treatment, they will be asked if they are still available for follow-up data collection.

\section{Trial exit}

Participants will exit the trial completely if:

- They have completed their participation

- They request to/are unable to continue being followed up

- They experience an $\mathrm{AE}$ (if the $\mathrm{AE}$ is deemed due to trial involvement) such that they are unable to complete follow-up

- They die

\section{Overall timescale for the study}

Recruitment began as soon as all necessary approvals (REC, Medicines and Healthcare products Regulatory Agency (MHRA) and Trust R\&D) were obtained. The intention is that recruitment will run until a power calculation of participant number is reached. A power calculation will be performed from ongoing cohort study and existing evidence with a maximum of 100 participants in total. Patients are involved in the trial for 12 weeks only.

The outline of events is given in Table 4 .

\section{Study completion}

Completion refers to the date of final data collection from the last patient. Paper records from the trial will be stored for 5 years from trial end.

Data will be collected and collated using a specifically designed database which will be kept on hospital central servers on a limited access hard drive. Access will be via password-protected log-in on hospital servers only and will be limited to members of the Academic Vascular Surgery Unit. The file itself will have password-protected 
Table 4 Standard Protocol Items: Recommendations for Interventional Trials (SPIRIT) diagram of events timeline

\begin{tabular}{|c|c|c|c|c|c|c|c|}
\hline \multirow[b]{3}{*}{ Timepoint } & \multicolumn{7}{|l|}{ Study period } \\
\hline & \multirow{2}{*}{$\begin{array}{l}\text { Enrolment and } \\
\text { screening }\end{array}$} & \multirow[t]{2}{*}{ Allocation } & \multicolumn{4}{|l|}{ Post randomisation } & \multirow[t]{2}{*}{ Exit } \\
\hline & & & Treatment period & $4 / 52 \mathrm{~F} / \mathrm{U}$ & $\begin{array}{l}8 / 52 \\
F / U \\
\end{array}$ & $\begin{array}{l}12 / 52 \\
F / U\end{array}$ & \\
\hline \multicolumn{8}{|l|}{ Enrolment: } \\
\hline Eligibility screen & $x$ & & & & & & \\
\hline Demographics & $x$ & & & & & & \\
\hline Past medical history & $x$ & & & & & & \\
\hline Provision of Patient Information Sheet & $x$ & & & & & & \\
\hline Informed consent & & $x$ & & & & & \\
\hline Randomisation/allocation & & $x$ & & & & & \\
\hline \multicolumn{8}{|l|}{ Interventions: } \\
\hline Active shockwave treatment (Group A) & & & $\begin{array}{l}9 \text { sessions } \\
(3 \times \text { per week for } 3 \text { weeks) }\end{array}$ & & & & \\
\hline Sham treatment (Group B) & & & $\begin{array}{l}9 \text { sessions } \\
(3 \times \text { per week for } 3 \text { weeks) }\end{array}$ & & & & \\
\hline \multicolumn{8}{|l|}{ Assessments: } \\
\hline Ankle-brachial pressure index & $x$ & & & $x$ & $x$ & $x$ & \\
\hline Claudication distance & $x$ & & & $x$ & $x$ & $x$ & \\
\hline Maximum walking distance & $x$ & & & $x$ & $x$ & $x$ & \\
\hline SF-36 questionnaire & $x$ & & & $x$ & $x$ & $x$ & \\
\hline EQ-5D questionnaire & $x$ & & & $x$ & $x$ & $x$ & \\
\hline Patient tolerability VAS & & & Recorded after each treatment & & & & \\
\hline Adverse events (bruising, bleeding, etc.) & & & Recorded after each treatment & $x$ & $x$ & $x$ & \\
\hline
\end{tabular}

opening. Electronic records will be stored for 5 years from trial end.

\section{Data management}

The use and control of all data will comply at all times with the requirements of the Data Protection Act 1998. The Principal Investigator of the study will register with the local Data Protection Officer. All data management procedures will be detailed and referred to as the Data Management Plan. All data will be entered and checked by a second person.

The database will be designed and built by the research team using Microsoft Excel. Appropriate CRFs will be prepared for the collection of the data requested by the protocol. All response variables will be entered into the database by the data management personnel.

\section{Statistical considerations}

Statistical advice has been sought from the Statistical Consultancy Service (Hull York Medical School, University of York) in preparing this protocol. Further review will be sought when all results have been collected and the analysis performed.
Data will be analysed as intention to treat. The SPSS computer package will be used with a $p$ value of $<0.05$ taken as the level of significance.

\section{Sample size calculation}

A power calculation will be performed from the ongoing cohort study and existing evidence with a maximum of 100 participants in total.

\section{Analysis}

Data will be analysed on an intention-to-treat basis. The main aim is to provide estimates of distribution and variability of outcomes with comparisons made between the shockwave treatment group and sham group. These will be expressed principally as means or medians and confidence intervals.

\section{Monitoring and quality assurance}

The Principal Investigator is responsible for the day-today monitoring and management of the study. The Research Office, on behalf of the Sponsor, will monitor and conduct random audits on a selection of studies in its clinical research portfolio. Monitoring and auditing will be conducted in accordance with the Department of 
Health Research Governance Framework for Health and Social Care (April 2005), and in accordance with the Sponsor's monitoring and audit policies and procedures.

The organisation, monitoring and quality assurance of this clinical study are the responsibility of the Sponsor and the Principal Investigator. To ensure the accuracy of data, direct access to source documents by the representatives of both the Sponsor and regulatory authorities is mandatory. Anonymity of the subjects will be maintained at all times.

The investigator(s)/institution(s) will permit studyrelated monitoring, audits, REC review and regulatory inspection(s), providing direct access to source data/documents. Patient consent to this is specifically sought in the Informed Consent Form.

\section{Study completion or discontinuation}

Upon completion of the study, the following activities, when applicable, must be conducted by the Investigator and by the Sponsor's Monitor, as appropriate:

- Data clarifications and/or resolutions

- Review of site study records for completeness

In addition, the Sponsor, the Funder and the Principal Investigator reserve the right to temporarily suspend or prematurely discontinue this study for any reason. If such action is taken, the Sponsor will discuss this with the Funder and the Investigator (including the reasons for taking such action) at that time.

If the study is terminated for safety reasons, the Sponsor will promptly inform the regulatory authorities of the suspension or termination of the study and the reason(s) for the action. The Investigator must inform the REC promptly and provide the reason for the suspension or termination. After such a decision, the Investigator must call in all participating patients within a reasonable time period. At this visit all medical files and CRFs must be completed as far as possible.

\section{Administrative procedures Ethical considerations}

This protocol has been prepared in accordance with the Standard Protocol Items: Recommendations for Interventional Trials (SPIRIT) checklist. See Additional file 1.

This study is conducted in accordance with the principles of the Declaration of Helsinki (Ethical Principles for Medical Research Involving Human Subjects).

\section{Participant data protection}

Participants will be informed that their data are held on file and that these data may be viewed by the Sponsor and by external auditors on behalf of either the Sponsor or regulatory agencies. Participants will similarly be informed that these data and a report of the study will be submitted to the Sponsor and may also be submitted to government agencies and perhaps for publication, but that they will only be identified in such reports by their study identification number, initials and perhaps their gender and age. The investigators undertake to hold all personal information in confidence and in compliance with the Data Protection Act 1998 and Caldicott Committee policies.

Data will be collected and collated using the database and data analysis software SPSS. This will be kept on hospital central servers on a limited access hard drive. Access will be via password-protected log-in on hospital servers only and will be limited to members of the Academic Vascular Surgery Unit. The file itself will have password-protected opening.

\section{Data handling and record keeping}

The Principal Investigator will be responsible for data collection, recording and quality. Electronic data will be stored on a National Health Service (NHS) Trust computer within the vascular laboratory. The IT services department has a backup procedure approved by auditors for disaster recovery. Servers are backed up to tape media each night; the tapes run on a 4-week cycle. Files stay on the server unless deleted by accident or deliberately. Anything deleted more than 4 weeks previously is therefore lost. Additional archive backups are taken for archived data, so data should not be lost from this type of system, e.g. with the use of FileVision, which stores medical records. Tapes are stored in a fireproof safe. Study documents (paper and electronic) will be retained in a secure location and kept locked when not in use during the trial and after it has finished. All essential documents including source documents will be retained for a minimum period of 5 years after study completion (last visit of the last patient). A label stating the date after which the documents can be destroyed will be placed inside the front cover of the case notes of trial participants. Data will be collected and retained in accordance with the Data Protection Act 1998.

\section{Indemnity}

This is an NHS-sponsored research study. If there is negligent harm during the clinical trial when the NHS body owes a duty of care to the person harmed, NHS indemnity covers NHS staff and medical academic staff with honorary contract only when the trial has been approved by the Hull and East Yorkshire R\&D department. NHS indemnity does not offer no-fault compensation and is unable to agree in advance to pay compensation for non-negligent harm. The University of Hull has an insurance policy that includes cover for no-fault compensation in respect of accidental injury to a research subject. 


\section{Discussion}

There is significant research suggesting a neovascularisation/angiogenic response to ESWT when applied to soft tissue. However, only several small, low-level research trials have investigated the use of ESWT in patients with peripheral arterial disease and, more specifically, intermittent claudication. Non-invasive management of patients suffering from intermittent claudication consists of best medical treatment plus supervised exercise programmes (SEPs). Unfortunately, uptake and adherence to SEPs is extremely poor in most centres; therefore, there is potential for an alternative non-invasive treatment option. The purpose of this study is to firstly investigate whether this treatment is safe and well tolerated by patients when applied to the calf muscle bulk, and also to study whether the angiogenic response seen in soft tissue research relating to ESWT has a positive effect on this cohort of patients and whether it is significant enough to improve clinical outcomes such as maximum walking distance, pain-free walking distance and ankle-brachial pressure index.

\section{Trial status}

This trial remains in the recruitment phase.

\section{Additional file}

Additional file 1: SPIRIT checklist. (DOC $121 \mathrm{~kb})$

\section{Abbreviations}

ABPI: Ankle-brachial pressure index; BMI: Body mass index; BMT: Best medical treatment; Cl: Confidence interval; CILLI: Clinical indicator of lower limb ischaemia; CLI: Critical limb ischaemia; CRP: C-reactive protein; ESWT: Extracorporeal shockwave therapy; GP: General practitioner; HDL: High-density lipoprotein; IC: Intermittent claudication; ICD: Intermittent claudication distance; NICE: National Institute of Health and Care Excellence; nM: Nanomole; P: Probability; PABPI: Post exercise ankle-brachial pressure index; PAD: Peripheral arterial disease; PF: Physical function; PRD: Patient reported walking distance; PTA: Percutaneous transluminal angioplasty; QoL: Quality of life; SEP: Supervised exercise programme; SF: Social function; SF-36: Short Form 36; TASC: Trans-Atlantic Inter-Society Consensus; VLDL: Very low-density lipoprotein; vWF: Von Willebrand factor
\end{abstract}

\section{Acknowledgements}

Full acknowledgement is given to the assistance and guidance from my academic senior colleagues (Smith, Carradice, Chetter) for their help and support in developing this trial protocol. Acknowledgment is given to Miss Amy Harwood in providing crucial support in terms of clinical assessments which are vital in the methodology of this trial.

\section{Funding}

The study is funded through the Academic Vascular Surgical Unit at Hull Royal Infirmary. Participants will not receive any financial incentive to take part in this study.

\section{Availability of data and materials}

The investigators and institution will permit monitoring, audits, REC and MHRA review where applicable and provide direct access to source data and documents.

\section{Authors' contributions}

$\mathrm{TC}$ led the instigation and setup of this trial as Principal Investigator. $\mathrm{AH}$ remained blinded to participant groups and provided all baseline and follow-up assessments. GS provided academic support and revised the manuscript. JT assumed responsibility for revision of the manuscript and author correspondence, and has joined the team as a study investigator. DC provided academic support and patient recruitment and contributed to the preparation of the manuscript. IC participated in the design and registration of the clinical trial and oversaw the preparation of the manuscript. All authors read and approved the final manuscript. No professional writers have been used in the preparation of this manuscript, nor will they be used in the preparation of any publications arising from this work.

\section{Competing interests}

The authors declare that they have no competing interests.

\section{Consent for publication}

All patients have provided consent for dissemination of results and information related to this trial. All authors and investigators in this trial give consent for dissemination.

The trial will be reported in accordance with the CONSORT statement (Consolidated Standards of Reporting Trials, www.consort-statement.org) with the anticipation of publication in a peer-reviewed journal. Publication will not be influenced by the funding company or Sponsor.

\section{Ethics approval and consent to participate}

Research Ethics Committee approval was granted in March 2015 (Reference: 14/ EE/1257). The approval body is NRES Committee East of England - Cambridge East. The Integrated Research Approval System (IRAS) project ID is 166137. Before enrolment into the study, participants will be fully informed of the nature of the study and all relevant aspects of study procedures. It is the investigators' responsibility to explain the nature of the study, its purpose and associated procedures, the expected duration and the potential benefits and risks of participation to each subject prior to their entry into the study (i.e. before examinations and procedures associated with selection for the study are performed).

Each prospective candidate will be provided with the Patient Information Sheet and Informed Consent Forms, to be compiled as one integrated document. Each subject will have ample opportunity to ask questions and will be informed about his/her right to withdraw from the study at any time without any disadvantage and without having to provide reasons for this decision.

Once the essential study information has been provided and the Investigator is assured that each individual patient understands the implications of participating in the study, the eligible patients who pass the screening evaluation will be recruited. Informed Consent Forms shall be signed and dated by the appropriate parties. A notation that written informed consent has been obtained will be made on the CRF and a record made in their clinical notes. Completed Informed Consent Forms will be retained by the Investigator, a copy entered into the clinical notes and a copy provided by the Investigator to the patient. The Informed Consent Form is included as part of the submission to the REC.

The Informed Consent Form and any other written information provided to subjects will be revised whenever important new information becomes available that may be relevant to the subject's consent, or there is an amendment to the protocol which necessitates a change to the content of the subject information and/or the written Informed Consent Form. The investigators will inform the subject of changes in a timely manner and will ask the subject to confirm their continued participation in the study by their signature on the revised Informed Consent Form. Any revisions to the written Informed Consent Form and written information will receive main REC approval in advance of use.

Patients will be free to withdraw at any time without affecting their healthcare or legal rights.

\section{Study sponsor}

Hull and East Yorkshire NHS Trust will act as the study Sponsor, as the study takes place on an NHS hospital site. They are responsible for the monitoring of the study. They have had no input into the study design or data collection. 
Received: 31 May 2016 Accepted: 16 February 2017

Published online: 06 March 2017

\section{References}

1. NICE clinical guideline 147. Lower limb peripheral arterial disease: diagnosis and management. August 2012. http:/guidance.nice.org.uk/cg147. Accessed 28 Feb 17.

2. Chetter IC, Spark JI, Dolan P, Scott DJ, Kester RC. Quality of life analysis in patients with lower limb ischaemia: suggestions for European standardisation. Eur J Vasc Endovasc Surg. 1997;13(6):597-604.

3. Shammas NW. Epidemiology, classification, and modifiable risk factors of peripheral arterial disease. Vasc Health Risk Manag. 2007;3(2):229-34. PMCID: PMC1994028.

4. Cassar K, Bachoo P, Ford I, Greaves M, Brittenden J. Markers of coagulation activation, endothelial stimulation and inflammation in patients with peripheral arterial disease. Eur J Vasc Endovasc Surg. 2005:29:171-6.

5. Schmieder FA, Comerota AJ. Intermittent claudication: magnitude of the problem, patient evaluation, and therapeutic strategies. Am J Cardiol. 2001; 87(suppl):3D-13D

6. Tsang GM, Sanghera K, Gosling P, Smith FC, Patterson IS, Simms MH, Shearman CP. Pharmacological reduction of the systemically damaging effects of local ischaemia. Eur J Vasc Surg. 1994;8(2):205-8.

7. Tisi PV, Shearman CP. Biochemical and inflammatory changes in the exercising claudicants. Vasc Med. 1998;3(3):189-98.

8. Turton EPL, Coughlin PA, Kester RC, Scott DJA. Exercise training reduces the acute inflammatory response associated with claudication. Eur J Vasc Endovasc Surg. 2002;23:309-16.

9. Mittermayr R, Antonic V. Extracorporeal shock wave therapy (ESWT) for wound healing: technology, mechanisms, and clinical efficacy. Wound Repair Regen. 2012;20(4):456-65.

10. Wang CJ. An overview of shock wave therapy in musculoskeletal disorders. Chang Gung Med J. 2003;26(4):220-32.

11. Schaden W, Thiele R. Shock wave therapy for acute and chronic soft tissue wounds: a feasibility study. J Surg Res. 2007;143(1):1-12. Epub 2007 Sep 27.

12. Wang CJ. Treatment of nonunions of long bone fractures with shock waves. Clin Orthop Relat Res. 2001;387:95-101.

13. Xu Z-H, Jiang Q. Extracorporeal shock wave treatment in nonunions of long bone fractures. Int Orthop. 2009;33(3):789-93. doi:10.1007/s00264-008-0553-8.

14. Elster EA. Extracorporeal shock wave therapy for nonunion of the tibia. $J$ Orthop Trauma. 2010;24(3):133-41. doi:10.1097/BOT.0b013e3181b26470.

15. Schaden W, Fischer A, Seiler A. Extracorporeal shock wave therapy of nonunion or delayed osseous union. Clin Orthop. 2001;387:90-4.

16. Frairia R, Berta L. Biological effects of extracorporeal shock waves on fibroblasts. A review. Muscles Ligaments Tendons J. 2011;1(4):138-47.

17. Mariotto S. Extracorporeal shock wave therapy in inflammatory diseases: molecular mechanism that triggers anti-inflammatory action. Curr Med Chem. 2009;16(19):2366-72.

18. Nishida T. Extracorporeal cardiac shock wave therapy markedly ameliorates ischemia-induced myocardial dysfunction in pigs in vivo. Circ Am Heart Assoc. 2004;110:3055-61.

19. Gutersohn A. Upregulation of vascular endothelial growth factor m-RNA in human umbilical vascular endothelial cells via shock waves. Eur J Heart Failure. 2000;2(S1):42.

20. Zhang $X$. The dose-effect relationship in extracorporeal shock wave therapy: the optimal parameter for extracorporeal shock wave therapy. J Surg Res. 2014;186(1):484-92.

21. Wang CJ. Shock wave therapy induces neovascularization at the tendonbone junction. A study in rabbits. J Orthop Res. 2003;21(6):984-9.

22. Fukumoto $Y$, Ito A. Extracorporeal cardiac shock wave therapy ameliorates myocardial ischemia in patients with severe coronary artery disease. Coron Artery Dis. 2006;17(1):63-70.

23. Ciccone MM, Notarnicola A. Shockwave therapy in patients with peripheral artery disease. Adv Ther. 2012;29(8):698-707. doi:10.1007/s12325-012-0038-4. Epub 2012 Aug 2.

24. Tara S, Miyamoto M. Low-energy extracorporeal shock wave therapy improves microcirculation blood flow of ischemic limbs in patients with peripheral arterial disease: pilot study. J Nippon Med Sch. 2014;81(1):19-27.

25. Belcaro G, Cesarone MR. Effects of shock waves on microcirculation, perfusion, and pain management in critical limb ischemia. Angiology. 2005;56(4):403-7.

26. Serizawa F, Ito K. Extracorporeal shock wave therapy improves the walking ability of patients with peripheral artery disease and intermittent claudication. Circ J. 2012;76(6):1486-93. Epub 2012 Mar 3.

\section{Submit your next manuscript to BioMed Central and we will help you at every step:}

- We accept pre-submission inquiries

- Our selector tool helps you to find the most relevant journal

- We provide round the clock customer support

- Convenient online submission

- Thorough peer review

- Inclusion in PubMed and all major indexing services

- Maximum visibility for your research

Submit your manuscript at www.biomedcentral.com/submit 